\title{
VARIAÇÃO NICTEMERAL DE PARÂMETROS ABIÓTICOS E CLOROFILA a EM UMA ESTAÇÃO OCEANOGRÁFICA NA PLATAFORMA CONTINENTAL DO AMAZONAS
}

\author{
MARIA DE LOURDES SOUZA SANTOS ${ }^{1}$ \& KÁTIA MUNIZ ${ }^{2}$ \\ 1 - Instituto Sócio Ambiental e dos Recursos Hídricos. Universidade Federal Rural da Amazônia. Av. Tancredo Neves, 2501. 66077-530. \\ Belém/PA - Brasil.mdelssantos@yahoo.com.br \\ 2 - Depto de Oceanografia - UFPE, Av. Arquitetura s/n, 50740-550. Recife/ PE. Brasil. katia@ufpe.br
}

\begin{abstract}
RESUMO
Durante as Operações Oceanográficas Norte III e Norte IV (Programa REVIZEE), foram coletadas amostras de água, durante 24 horas, em intervalo de três em três horas, nos dias 20 de maio de 1999 (período de descarga máxima do rio Amazonas) e 13 de agosto de 2001 (período de transição do rio Amazonas), em uma estação localizada na Plataforma Continental do Amazonas. O objetivo deste trabalho foi avaliar a variação de parâmetros abióticos e clorofila a, obtidos durante essa coleta. No dia 13 de agosto de 2001, foram observadas as menores variações de salinidade (de 34,0 a 36,4 ) e as menores concentrações de nitrato (média de 1,7 mmol. $\mathrm{L}^{-1}$ ) e fosfato (média de $0,2 \mathrm{mmol} . \mathrm{L}^{-1}$ ), e valores elevados de clorofila a (máximo de 5,47 mg. $\mathrm{m}^{-3}$ ). Enquanto, no dia 20 de maio de 1999, foram detectadas as maiores variações de salinidade (de 20,0 a 35,6), as maiores concentrações de nitrato (média de $4,9 \mathrm{mmol} . \mathrm{L}^{-1}$ ), de fosfato (média de $0,2 \mathrm{mmol} . \mathrm{L}^{-1}$ ), e os menores valores de clorofila a (máximo de $0,32 \mathrm{mg}^{-3} \mathrm{~m}^{-3}$ ), o que indica ser a transparência da água o fator condicionante para o desenvolvimento da produtividade primária, na área da Plataforma Continental do Amazonas.
\end{abstract}

PALAVRAS-CHAVE: Plataforma Continental do Amazonas, nutrientes, clorofila a.

\section{ABSTRACT \\ Nictemeral variation in an oceanography station in the Amazon continental shelf}

\begin{abstract}
During the Operations Oceanography's North III and North IV (program REVIZEE), samples of water were collected for 24 hours, in a period by three hours, on May 20, 1999 (period of maximum discharge of the Amazon's river) and August 13, 2001 (transition period of the Amazon's river), in a located station in the Amazon Continental Shelf. The objective of this work was to evaluate the variation of parameters abiotics and chlorophyll a, during that nictemeral collection. On August 13, 2001, the smallest salinity variations (of 34,0 and 36,4 ) and the smaller concetrations of nitrate (medium of $1,7 \mu \mathrm{mol}^{-1} \mathrm{~L}^{-1}$ ) and of phosphate (medium of $0,2 \mu \mathrm{mol} . \mathrm{L}^{-1}$ ) were detected and the largest chlorophyll a concetrations (maximum of $5,47 \mathrm{mg}, \mathrm{m}^{-3}$ ). While on May 20 , 1999, the largest concentrations of nitrate were detected (medium of $4,9 \mu \mathrm{mol} . \mathrm{L}^{-1}$ ) and phosphate (medium of $0,4 \mu \mathrm{mol} . \mathrm{L}^{-1}$ ), and largest variations of salinity (minimum of 20,0 and maximum of 35,6 ) and concentrations of chlorophyll a (maximum of 0,32 $\mathrm{mg} \cdot \mathrm{m}^{-3}$ ), that indicates be the transparency of the water the limiting factor for the development of the primary productivity, in the area of the Amazon Continental Shelf.
\end{abstract}

KEYWORDS: Amazon Continental Shelf, nutrients, chlorophyll a.

\section{INTRODUÇÃO}

O rio Amazonas é considerado o maior rio do mundo, em termos de descarga de água (100.000$\left.220.000 \mathrm{~m}^{3} \mathrm{~s}^{-1}\right)$ e de sedimentos $\left(11-13 \times 10^{8}\right.$ ton.ano ${ }^{-1}$ ) (Kineke et al., 1996), com um fluxo de componentes dissolvidos de $2-3 \times 10^{8}$ ton.ano $^{-1}$ (Gibbs, 1972), e uma variabilidade intra-anual, que ocorre sazonalmente com mínimo $\left(0,9 \times 10^{5} \mathrm{~m}^{3} \mathrm{~s}^{-1}\right)$, de outubro a dezembro, e máximo $\left(2,3 \times 10^{5} \mathrm{~m}^{3} \mathrm{~s}^{-1}\right)$, de maio a junho (Curtin \& Legeckis, 1986).

A imensa descarga do rio Amazonas não permite a entrada de água do mar na foz do rio (mesmo durante o período de descarga mínima), e é responsável pelo desenvolvimento de um meio altamente dinâmico na sua foz, o que propicia o estabelecimento da maior zona estuarina do mundo (Nittrouer et al., 1991).

A literatura registra vários trabalhos oceanográficos a respeito do rio Amazonas: Ryther et al. (1967) determinaram o tamanho e a localização da área influenciada por esse rio e os níveis dos nutrientes e das populações planctônicas.
Teixeira \& Tundisi (1967) verificaram uma diminuição progressiva na produção primária, no número de organismos por litro entre a área costeira e a oceânica, e também uma mudança dos diferentes grupos fitoplanctônicos.

Diégues (1972) descreveu a extensão da influência do rio Amazonas sobre o Oceano Atlântico; classificou a foz desse rio como um estuário positivo, em que a evaporação é menor que a soma das precipitações e contribuição de rios, e, sob o ponto de vista geomorfológico, como um estuário plano costeiro, por possuir fundos rasos, de declives suaves, com a profundidade crescendo mais ou menos uniformemente em direção ao oceano.

Posteriormente, trabalhos foram desenvolvidos, como DeMaster et al. (1983) e DeMaster et al. (1986), que avaliaram a distribuição do silicato na Plataforma Continental do Amazonas. Fox et al. (1986) descreveram quantitativamente 0 papel dos sedimentos no controle das concentrações de fósforo inorgânico solúvel no estuário do rio Amazonas. 
Santos (2004) estudou a distribuição das formas orgânicas $e$ inorgânicas de fósforo e nitrogênio, na coluna de água dessa Plataforma, e sua relação com a produtividade primária.

Contudo, percebe-se não haver informações da dinâmica de parâmetros abióticos e clorofila $a$, em estações pontuais, dos tipos nictemerais (atividade cíclica de 24 horas), na Plataforma Continental do Amazonas.

Teixeira \& Gaeta (1991) relatam que um dos aspectos fundamentais para a obtenção de dados, com maior significado sobre a produção primária de um ecossistema e para confecção de modelos, está relacionado aos conhecimentos obtidos a partir da determinação da periodicidade nictemeral, não só do fitoplâncton, como também das características ambientais.
Dentro desse contexto, este trabalho teve por objetivo avaliar a variação nictemeral de parâmetros abióticos (temperatura, salinidade, oxigênio dissolvido, taxa de saturação de oxigênio dissolvido, $\mathrm{pH}$, nitrato, nitrito, $\mathrm{N}$-amoniacal, fosfato, silicato) e a clorofila $a$, em uma estação oceanográfica na Plataforma Continental do Amazonas.

\section{MATERIAL E MÉTODOS}

Amostras de água foram coletadas durante 24 horas, em intervalo de três em três horas, a bordo do Navio Oceanográfico "ANTARES", da Marinha do Brasil, em uma estação localizada na Plataforma Continental do Amazonas (figura 1), latitude $48^{\circ} 45,60^{\prime} \mathrm{W}$ e longitude $2^{\circ} 5,40^{\prime} \mathrm{N}$.

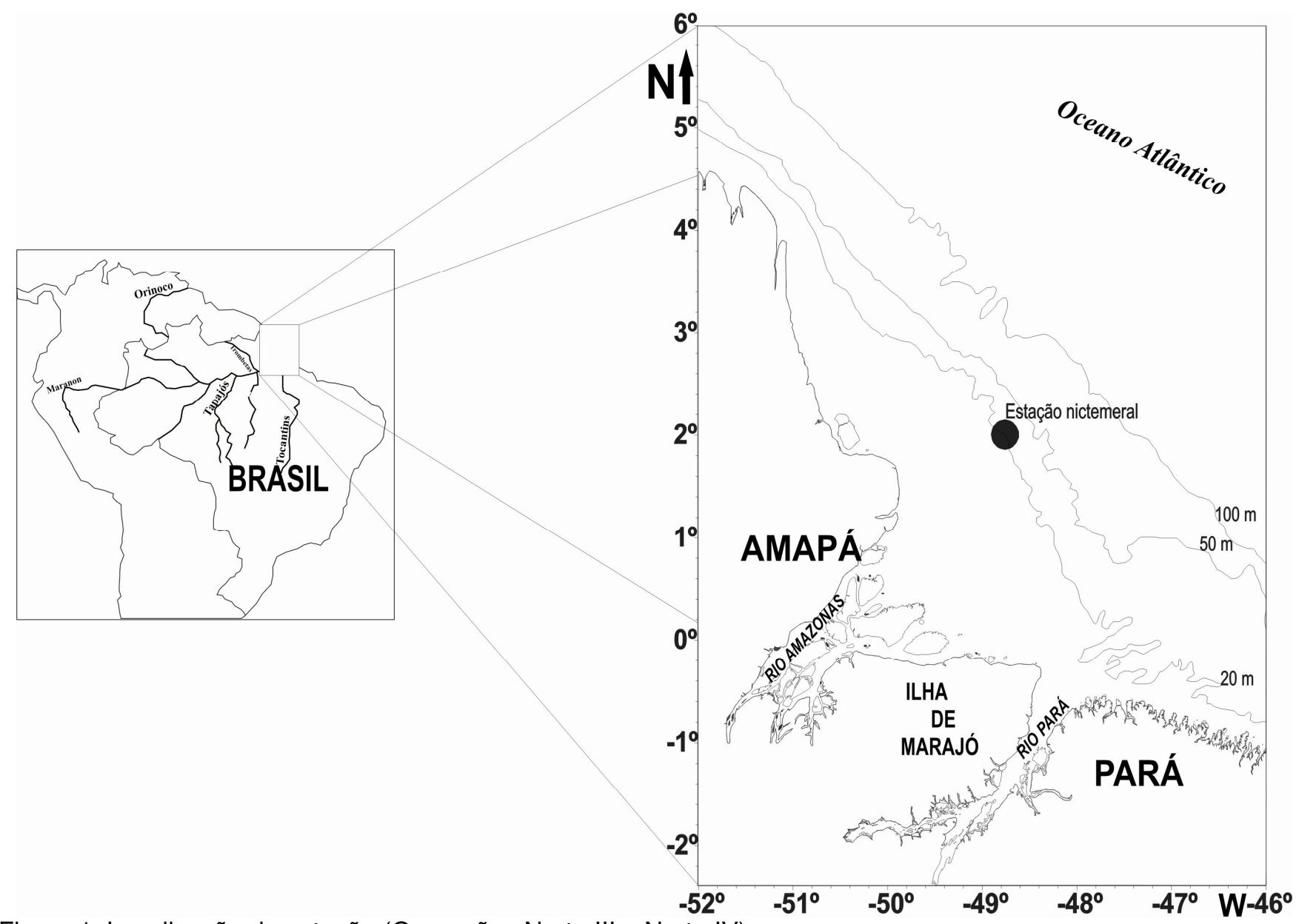

Figura 1: Localização da estação (Operações Norte III e Norte IV).

As coletas foram realizadas no do âmbito do Programa "Avaliação do Potencial Sustentável de Recursos Vivos na Zona Econômica Exclusiva" REVIZEE, durante as campanhas denominadas
Operação Norte III e Operação Norte IV, nos períodos de descarga máxima do rio Amazonas (no dia 20 de maio de 1999) e período de transição desse rio (no dia 13 de agosto de 2001), respectivamente. 
As coletas hidrológicas foram feitas com auxílio de garrafas de Niskin, em três profundidades: superfície $(1 \mathrm{~m}), 10 \mathrm{~m}$ e $20 \mathrm{~m}$. Desta forma foi possível abranger toda a coluna de água, visto que a profundidade local era de $26 \mathrm{~m}$.

Os seguintes dados foram obtidos a bordo do navio: (i) temperatura e salinidade registradas por um CTD (Conductivity, Temperature, Depth) SeaBird SBE 9-plus, ligado à unidade de bordo SBE 11, acoplado a uma Rosette; (ii) oxigênio dissolvido utilizando-se o método de Winkler modificado, descrito em Strickland \& Parsons (1972); (iii) taxa de saturação do oxigênio dissolvido, utilizando a International Oceanographic Tables (UNESCO, 1973); (iv) pH medido com um pHmetro da marca Hanna.

Para determinação do nitrito, do nitrato, do Namoniacal, do fosfato e do silicato, as amostras de água foram filtradas com filtros $G F / F$, da marca Whatman, armazenadas em frascos de polietileno de $500 \mathrm{~mL}$, e conservadas em freezer.

Posteriormente, no Laboratório de Oceanografia da Universidade Federal do Pará, as amostras de água foram descongeladas e as análises dos nutrientes (nitrito, nitrato, $\mathrm{N}$-amoniacal, fosfato e silicato) seguiram a metodologia descrita em Grasshoff et al. (1983). No laboratório, a água utilizada para determinação dos nutrientes era proveniente de um ALPHA Q.
Para a análise da clorofila $a$, a coleta foi realizada apenas na superfície, as análises foram processadas no Laboratório de Produtividade Primária, do Departamento de Oceanografia, da Universidade Federal de Pernambuco, de acordo com o método descrito em Teixeira (1971).

Os dados de salinidade, temperatura, $\mathrm{pH}$, oxigênio dissolvido (OD), taxa de saturação de oxigênio dissolvido, nitrito, nitrato, $\mathrm{N}$-amoniacal, fosfato, silicato e clorofila a foram analisados pelos métodos de estatística descritiva básica.

\section{RESULTADOS E DISCUSSÃO}

A variação nictemeral da temperatura da água teve diferenças pouco significativas nos períodos estudados (tabela 1). A média foi de $28,1 \pm 0,3^{\circ} \mathrm{C}$ (20 de maio de 1999) e de $28,2 \pm 0,2^{\circ} \mathrm{C}$ (13 de agosto de 2001). Na coluna de água, observou-se uma massa de água sem variações térmicas acentuadas ao longo de um período de 24 horas, entre as profundidades estudadas.

Ryther et al. (1967) descreveram que a temperatura superficial da água é quase constante, durante $\mathrm{O}$ ano (variando entre $28^{\circ} \mathrm{C}$ a $30^{\circ} \mathrm{C}$ ), na Plataforma Continental do Amazonas. Trabalhos realizados por Santos (2000), Santos \& Silva (2000), ratificaram a pequena oscilação de temperatura existente na área dessa Plataforma e área oceânica adjacente.

Tabela 1: Dados abióticos e de clorofila a (Número de dados - №, mínimo, máximo, média \pm desvio padrão - DP) obtidos durante uma coleta nictemeral, em uma estação oceanográfica localizada na Plataforma Continental do Amazonas, nos dias 20 de maio de 1999 e 13 de agosto de 2001.

\begin{tabular}{|c|c|c|c|c|c|c|c|c|}
\hline & \multicolumn{3}{|c|}{20 de maio de 1999} & \multicolumn{5}{|c|}{13 de agosto de 2001} \\
\hline & № & Mínimo & Máximo & Média $\pm \mathrm{DP}$ & № & Mínimo & Máximo & Média $\pm \mathrm{DP}$ \\
\hline Temperatura $\left({ }^{\circ} \mathrm{C}\right)$ & 27 & 27,7 & 28,6 & $28,1 \pm 0,3$ & 27 & 27,8 & 28,5 & $28,2 \pm 0,1$ \\
\hline Salinidade & 27 & 20,0 & 35,6 & $30,6 \pm 5,4$ & 27 & 34,0 & 36,4 & $36,1 \pm 0,5$ \\
\hline $\mathrm{OD}\left(\mathrm{ml} \cdot \mathrm{L}^{-1}\right)$ & 27 & 3,0 & 5,5 & $4,1 \pm 0,8$ & 26 & 4,1 & 4,9 & $4,4 \pm 0,2$ \\
\hline OD (\%) & 27 & 67,2 & 114,4 & $89,4 \pm 14,6$ & 26 & 103,0 & 124,0 & $111,0 \pm 5,7$ \\
\hline $\mathrm{pH}$ & 27 & 8,0 & 8,3 & $8,1 \pm 0,1$ & 27 & 7,7 & 7,8 & $7,8 \pm 0,01$ \\
\hline Nitrato ( $\left.\square \mathrm{mol} . \mathrm{L}^{-1}\right)$ & 27 & 3,5 & 6,9 & $4,9 \pm 0,6$ & 26 & 1,0 & 2,6 & $1,7 \pm 0,4$ \\
\hline Nitrito ( $\square$ mol. $\mathrm{L}^{-1}$ ) & 26 & 0,01 & 0,4 & $0,1 \pm 0,08$ & 26 & 0,1 & 0,6 & $0,4 \pm 0,1$ \\
\hline $\mathrm{N}$-amoniacal $\left(\square \mathrm{mol}^{-\mathrm{L}^{-1}}\right)$ & 27 & 0,04 & 0,5 & $0,2 \pm 0,1$ & 23 & 0,05 & 0,2 & $0,1 \pm 0,03$ \\
\hline Fosfato ( $\square \mathrm{mol} . \mathrm{L}^{-1}$ ) & 27 & 0,3 & 0,5 & $0,4 \pm 0,05$ & 26 & 0,09 & 0,2 & $0,2 \pm 0,05$ \\
\hline Silicato ( $\square \mathrm{mol} . \mathrm{L}^{-1}$ ) & 27 & 4,4 & 45,1 & $14,9 \pm 11,2$ & 26 & 2,4 & 4,7 & $3,5 \pm 0,6$ \\
\hline Clorofila a (mg.m $\left.{ }^{-3}\right)$ & 09 & 0,09 & 0,32 & $0,20 \pm 0,10$ & 09 & 0,87 & 5,47 & $2,31 \pm 1,34$ \\
\hline
\end{tabular}


A presença de águas oriundas do rio Amazonas foi indicada pelos menores valores salinidade. Na figura 2, observa-se uma variação maior no dia 20 de maio de 1999 (mínimo de 20,0 e máximo de 35,6 , média de $30,6 \pm 5,4$, tabela 1 ), período de descarga máxima desse rio, com a presença de águas oriundas desse rio na camada superficial. No dia 13 de agosto de 2001, variação foi menor (mínimo de 34,0 e máximo de 36,4, média de $36,1 \pm 0,5$, tabela 1 ), e foi verificada a presença de águas oceânicas em todas as profundidades estudadas.

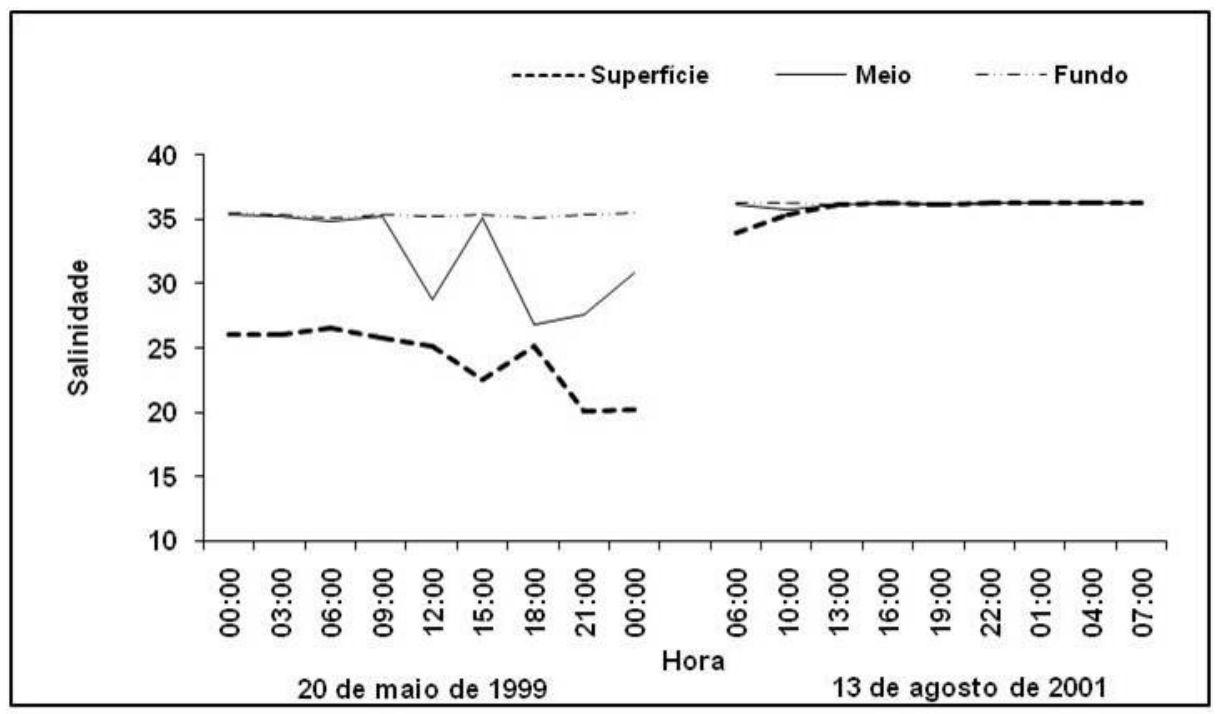

Figura 2: Variação nictemeral de salinidade em uma estação oceanográfica, na Plataforma Continental do Amazonas, nos dias 20 de maio de 1999 e 13 de agosto de 2001.

A presença de águas oceânicas e de águas oriundas do rio Amazonas, naquela Plataforma, foi verificada por vários autores (Geyer \& Kineke, 1995; Lentz \& Limeburner, 1995; DeMaster et al., 1996). A mistura da água do rio com a do oceano na plataforma continental foi também observada por Santos (2004), durante o período de menor descarga do rio Amazonas, porém com a presença de água oceânica nas zonas eufótica e afótica.

Com relação ao teor de OD e sua taxa de saturação, a média foi de 4,1 $\pm 0,8 \mathrm{ml}^{-\mathrm{L}^{-1}}$ (mínimo de 3,0 ml. $\mathrm{L}^{-1}$ e máximo de $5,5 \mathrm{ml}^{-\mathrm{L}^{-1}}$, tabela 1 ) e de 89,4 $\pm 14,6 \%$ (mínimo de $67,2 \%$ e máximo de $114,4 \%$, tabela 1) no dia 20 de maio de 1999; e de 4,4 $\pm 0,2$ $\mathrm{ml} . \mathrm{L}^{-1}$ (mínimo de $4,1 \mathrm{ml} . \mathrm{L}^{-1}$ e máximo de $4,9 \mathrm{ml} . \mathrm{L}^{-1}$, tabela 1) e de $111,0 \pm 5,7 \%$ (mínimo de 103,0\% e máximo de $124,0 \%$, tabela 1) no dia 13 de agosto de 2001, respectivamente.

A figura 3 mostra a variação nictemeral do oxigênio dissolvido (OD) durante os períodos estudados. A maior variação ocorreu no dia 20 de maio de 1999 e coincidiu com a variação de salinidade, quando foi possível verificar o maior valor de OD na superfície e uma diminuição das concentrações em direção ao fundo. 


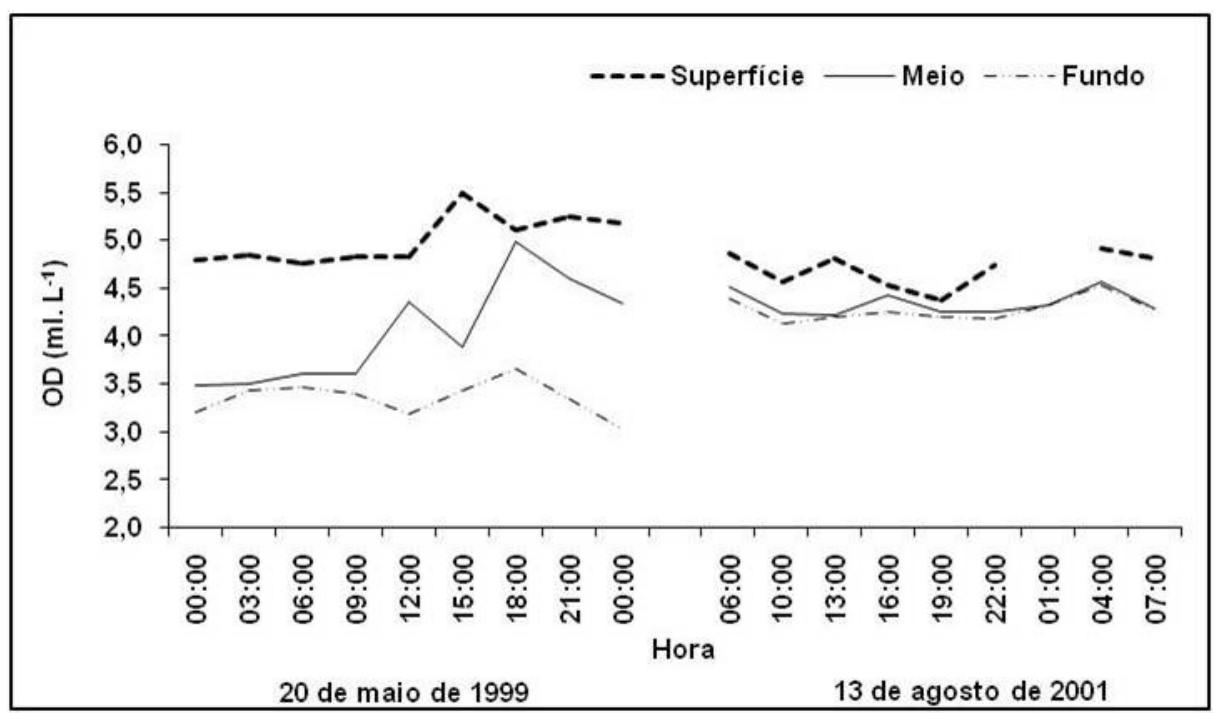

Figura 3: Variação nictemeral de $\mathrm{OD}\left(\mathrm{ml}^{\mathrm{L}} \mathrm{L}^{-1}\right)$ em uma estação oceanográfica, na Plataforma Continental do Amazonas, nos dias 20 de maio de 1999 e 13 de agosto de 2001.

As variações observadas podem ser explicadas pela decomposição da matéria orgânica, que consome o OD, e pelo processo fotossintético que libera esse gás para a coluna de água. $\mathrm{Na}$ área em estudo, altos valores de material em suspensão são esperados, como consequência da presença das águas oriundas do rio Amazonas juntamente com processos oceanográficos como marés, ondas e turbulências vertical, que favorecem a ressuspensão. Esses processos foram descritos por DeMaster et al. (1996) como uma fonte de silicato e ntrogênio inorgânico na plataforma externa.

Santos (2000) relacionou os valores de supersaturação de OD (taxa de saturação de 132,6\%) com as altas concentrações de biomassa fitoplanctônica $\left(9,79 \quad \mathrm{mg} \cdot \mathrm{m}^{-3}\right)$ na Plataforma Continental do Amazonas. No presente trabalho, valores de supersaturação de OD (tabela 1), os quais coincidiram com os máximos valores de OD, também foram detectados nos dois dias estudados.

Para o pH, o valor mínimo foi de 8,0 e o máximo de 8,3, no dia 20 de maio de 1999; e mínimo de 7,7 e máximo de 7,8 , no dia 13 de agosto de 2001, tabela 1 , sem variações acentuadas entre os períodos estudados e durante os horários de coleta, registrando-se apenas valores alcalinos em toda a coluna de água, com valores dentro dos limites considerados por Angeli (1979) como favoráveis à vida aquática $(6,0<\mathrm{pH}<8,5)$.
A pequena diferença observada entre os dias estudados pode ser relacionada ao sistema dióxido de carbono no ambiente marinho, que funciona como tampão e mantém $\mathrm{opH}$ em um limite estreito. $\mathrm{O}$ aumento da concentração de $\mathrm{CO}_{2}$ na água diminui o $\mathrm{pH}$ e a formação dos carbonatos e bicarbonatos tendem a aumentar o $\mathrm{pH}$. Por se tratar de um sistema de par ácido-base conjugado fraco consegue manter o pH dentro de uma faixa boa para a sobrevivência dos organismos.

A maior concentração de silicato foi detectada no dia 20 de maio de 1999 (mínimo de 4,4 $\square$ mol. $^{-1}$ e máximo de $45,1 \square$ mol.L $^{-1}$, tabela 1). Nesse dia foi possível observar uma inversão na concentração desse nutriente, com as maiores concentrações na superfície e menores no fundo (figura 4), o que pode ser explicado pelo volume da descarga do rio Amazonas, causando a elevação do teor de silicato nas águas superficiais e intermediárias.

Valores elevados para o silicato são esperados, já que, de acordo com DeMaster et al. (1983), o suprimento de sílica para o ambiente marinho na Plataforma Continental do Amazonas é de aproximadamente $4,9 \times 10^{13} \mathrm{~g} \mathrm{SiO}_{2}$ por ano.

Com a redução das águas oriundas do rio Amazonas, na Plataforma Continental do Amazonas, no dia 13 de agosto de 2001, as concentrações do silicato foram menores que no período anterior (mínimo de 2,4 $\square$ mol.L ${ }^{-1}$ e máximo de $4,7 \square$ mol.. $L^{-1}$, tabela 1 e figura 4 ). 


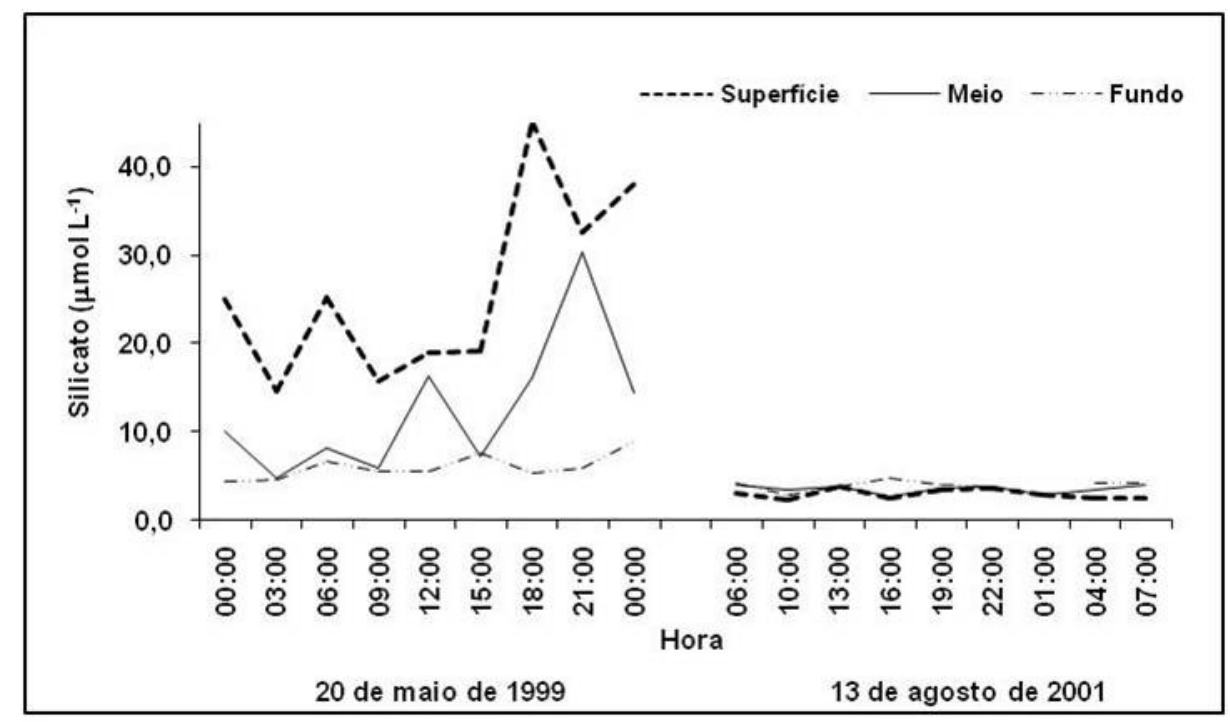

Figura 4: Variação nictemeral de silicato $\left(\square \mathrm{mol}^{-\mathrm{L}^{-1}}\right.$ ) em uma estação oceanográfica, na Plataforma Continental do Amazonas, nos dias 20 de maio de 1999 e 13 de agosto de 2001.

O nitrato (mínimo de $1,0 \square$ mol. $\mathrm{L}^{-1}$ e máximo de $2,6 \square \mathrm{mol}^{-L^{-1}}$, tabela 1 e figura 5) e o fosfato (mínimo de $0,1 \square$ mol. $\mathrm{L}^{-1}$ e máximo de $0,2 \square \mathrm{mol} . \mathrm{L}^{-1}$, tabela $1 \mathrm{e}$ figura 6) também apresentaram uma diminuição na concentração, no dia 13 de agosto de 2001, período de baixa vazão do rio Amazonas.

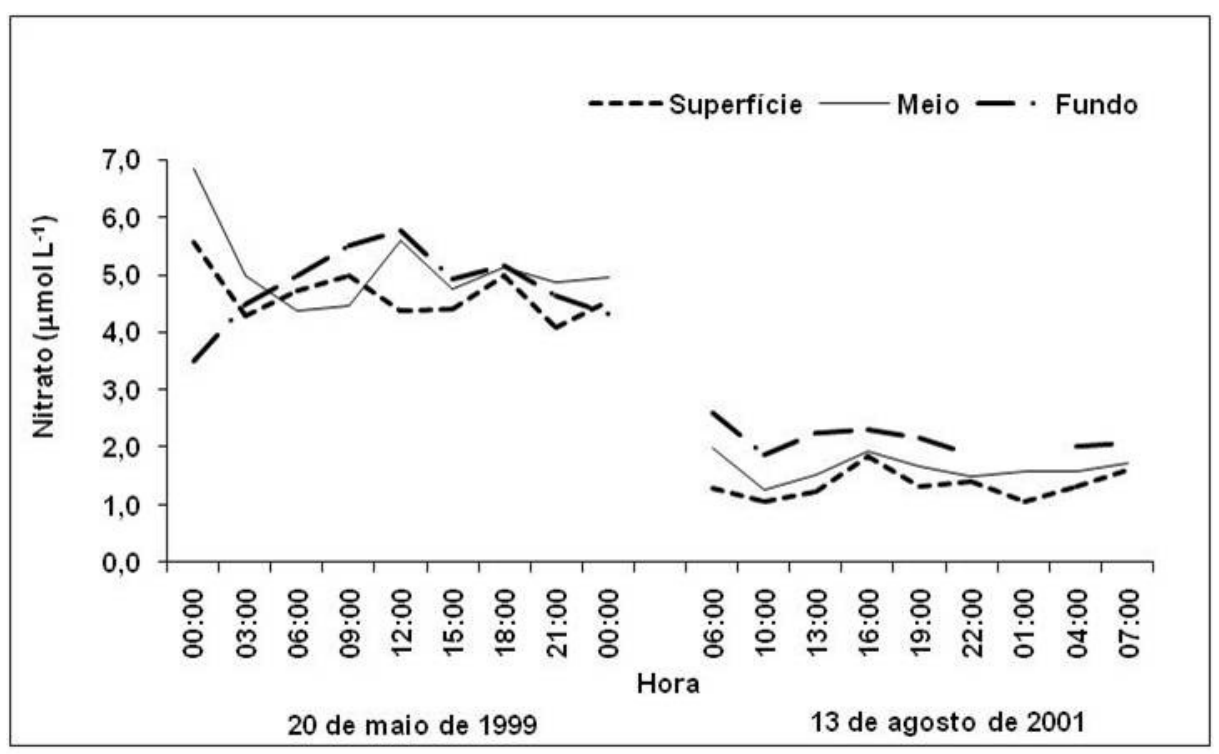

Figura 5: Variação nictemeral de nitrato $\left(\square \mathrm{mol}^{\mathrm{L}} \mathrm{L}^{-1}\right)$ em uma estação oceanográfica, na Plataforma Continental do Amazonas, nos dias 20 de maio de 1999 e 13 de agosto de 2001. 


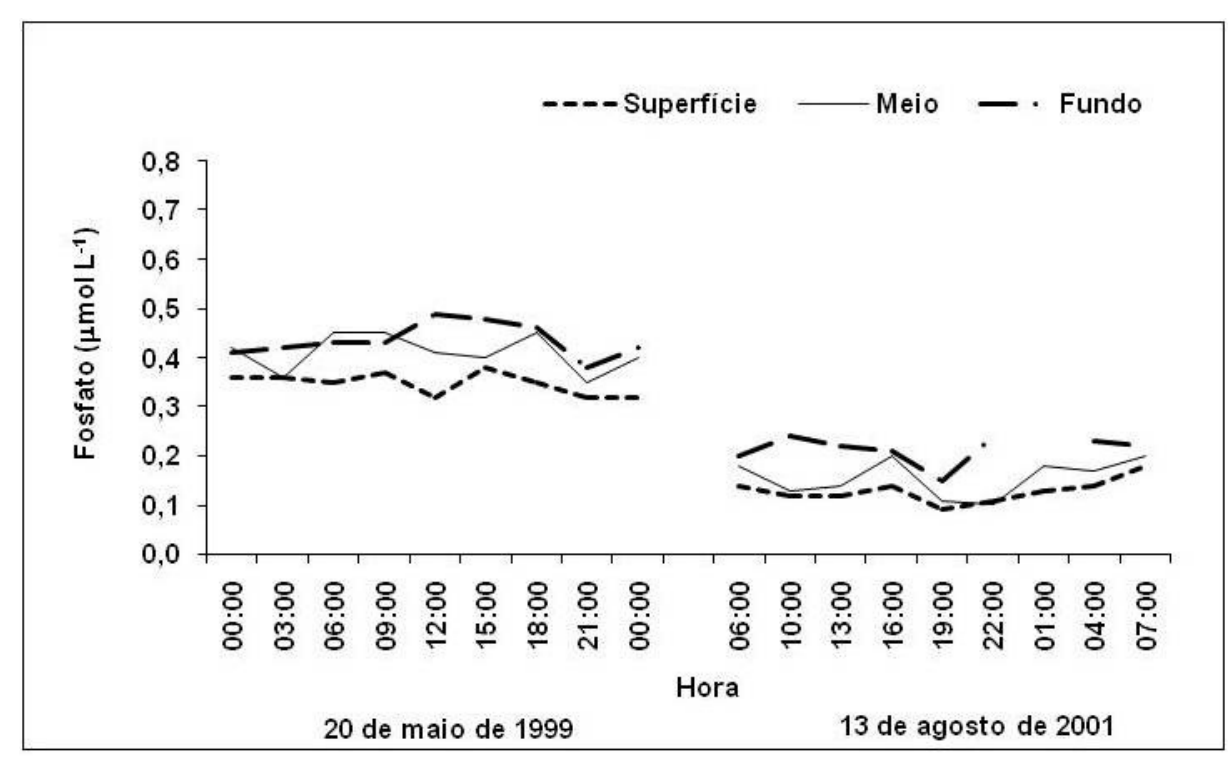

Figura 6: Variação nictemeral de fosfato $\left(\square \mathrm{mol} . \mathrm{L}^{-1}\right)$ em uma estação oceanográfica, na Plataforma Continental do Amazonas, nos dias 20 de maio de 1999 e 13 de agosto de 2001.

As distribuições do nitrato e fosfato foram semelhantes, com uma pequena variação entre a superfície e o fundo. Santos (2000) descreveu esse padrão de distribuição para esses nutrientes e verificou a diminuição em suas concentrações na área oceânica adjacente à Plataforma Continental do Amazonas.

DeMaster \& Pope (1996) e Santos (2000) descreveram o nitrogênio atuando como fator limitante para a biomassa fitoplanctônica na área da Plataforma Continental do Amazonas, sendo o nitrato a forma de nitrogênio inorgânica dissolvida predominante, com concentrações mais elevadas.

No presente trabalho o nitrato teve os maiores valores, seguido pelo $\mathrm{N}$-amoniacal (mínimo de 0,04 $\square$ mol. $\mathrm{L}^{-1}$ e máximo de $0,5 \square \mathrm{mol} . \mathrm{L}^{-1}$, dia 20 de maio de 1999; e mínimo de 0,05 $\square$ mol.L $^{-1}$ e máximo de $0,2 \square \mathrm{mol}^{-\mathrm{L}^{-1}}$, dia 13 de agosto de 2001, tabela 1) e nitrito (mínimo de 0,01 $\square \mathrm{mol}^{-1} \mathrm{~L}^{-1}$ e máximo de 0,4 $\square$ mol.. $\mathrm{L}^{-1}$, dia 20 de maio de 1999; e mínimo de $0,1 \square \mathrm{mol} \cdot \mathrm{L}^{-1}$ e máximo de $0,6 \square \mathrm{mol} \cdot \mathrm{L}^{-1}$, dia $13 \mathrm{de}$ agosto de 2001, tabela 1).
Como o nitrito é um elemento intermediário entre $\circ \mathrm{N}$-amoniacal e 0 nitrato, não foi observada uma variação nictemeral semelhante a do nitrato e fosfato. Em relação aos valores mínimos de $\mathrm{N}$ amoniacal, estes podem ser associados ao resultado da fácil assimilação pelo fitoplâncton e pelo processo de nitrificação.

As maiores concentrações de $\mathrm{N}$-amoniacal foram detectadas no período de maior descarga do rio Amazonas, no dia 20 de maio de 1999. Corroborando ao verificado por Santos (2000), que descreveu valores elevados dessa forma nitrogenada, durante o período de descarga máxima desse rio (máximo de 0,9 $\square$ mol.L').

Os menores valores de clorofila a (figura 7) foram detectados no dia 20 de maio de 1999 (mínimo de 0,09 mg.m-3 e máximo de 0,32 mg.m-3, tabela 1), na camada superficial, período de máxima descarga do rio Amazonas. Provavelmente conseqüência da alta concentração de material em suspensão, que diminui a transparência na coluna de água. 


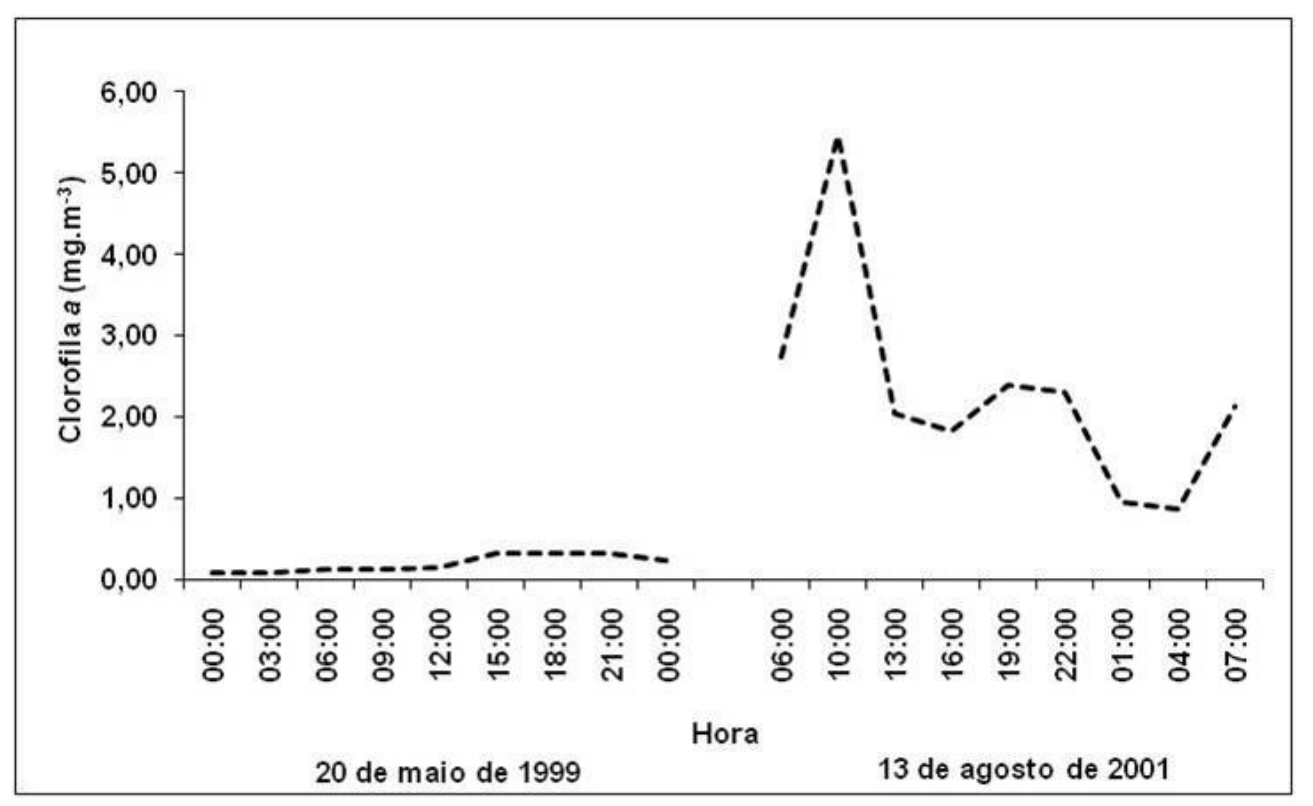

Figura 7: Variação nictemeral de clorofila a nas águas superficiais em uma estação oceanográfica, na Plataforma Continental do Amazonas, nos dias 20 de maio de 1999 e 13 de agosto de 2001.

Smith \& Russel (1995) relataram que o crescimento do fitoplâncton é determinado por três características na pluma do Amazonas: transparência, estratificação e a disponibilidade de nutrientes.

No dia 13 de agosto de 2001, foi observado um aumento na concentração de clorofila a (mínimo de 0,87 $\mathrm{mg} \cdot \mathrm{m}^{-3}$ e máximo de $5,47 \mathrm{mg} \cdot \mathrm{m}^{-3}$, tabela 1$)$ na camada superficial, no horário das dez horas da manhã.

Santos (2004) verificou que a distribuição superficial da clorofila a, no período da diminuição da descarga do rio Amazonas, foi influenciada pela dinâmica dos processos oceanográficos atuantes como, por exemplo, o transporte de massas de água na Plataforma Continental do Amazonas em direção noroeste, por meio da Corrente Norte do Brasil.

Santos (2000) caracterizou essa Plataforma como um ambiente eutrófico, com base em dados de clorofila $a$ entre $0,03 \mathrm{mg} \cdot \mathrm{m}^{-3}$ e $9,79 \mathrm{mg} \cdot \mathrm{m}^{-3}$. Santos (2004) encontrou valor de mediana da clorofila a de $1,67 \mathrm{mg} \cdot \mathrm{m}^{-3}$, mínimo de $0,13 \mathrm{mg} \cdot \mathrm{m}^{-3}$ e máximo de $41,45 \mathrm{mg} \cdot \mathrm{m}^{-3}$. De acordo com Santos (2004), a fertilidade da área foi favorecida durante a época da diminuição da descarga do rio Amazonas, com o valor da mediana próximo ao verificado por Santos (2000) e com os máximos valores acentuados observados em direção noroeste da desembocadura do rio Amazonas. Portanto, ratifica o descrito por Humborg
(1997) e Santos (2000), ou seja, na Plataforma Continental do Amazonas 0 aumento da produtividade primária é pontual e ocorre onde a concentração de nutrientes e a penetração de luz são favoráveis ao desenvolvimento fitoplanctônico.

Flores Montes et al. (1998) em estudo nas Barras Orange e Catuama do Canal de Santa Cruz, Itamaracá-PE, descreveram a variação nictemeral do fitoplâncton como bastante irregular e influenciada por diversos aspectos, principalmente pelos períodos de insolação, uma vez que, de uma forma geral, as maiores densidades foram determinadas nos horários diurnos, seguido pelo fluxo das marés e condições meteorológicas.

No presente trabalho, a variação nictemeral superficial de clorofila a foi favorecida no dia 13 de agosto de 2001, nesse dia, foram observadas maiores concentrações de clorofila a, com uma diminuição nas concentrações do nitrato e fosfato, nessa camada. A variação nictemeral de clorofila a teve aumento no horário das 10:00.

Foi possível avaliar que, apesar do aumento das concentrações dos nutrientes no dia 20 de maio de 1999 (período de máxima descarga do rio Amazonas), outro fator reduz a atividade dos organismos fitoplanctônicos, a turbidez. Ratificando o verificado por Humborg (1997) e Santos (2000). 


\section{CONCLUSÕES}

A variação nictemeral da clorofila a demonstrou a dinâmica existente na Plataforma Continental do Amazonas, por meio da influência do período da descarga do rio Amazonas, que condiciona a produtividade primária da estação estudada. A salinidade $e$ as concentrações de nutrientes influenciaram diretamente a clorofila $a$, desde que a transparência proporcionasse boas condições para o desenvolvimento fitoplanctônico. Em relação aos

\section{AGRADECIMENTOS}

Ao Conselho Nacional de Desenvolvimento Científico e Tecnológico (CNPq). À tripulação do Navio Oceanográfico ANTARES (H40), pelo apoio e atenção recebidos no trabalho de

\section{LITERATURA CITADA}

ANGELI, N. 1979. Influencia de la Polucion del agua sobre los elementos del plancton. In: PERSON, P. (ed.). La Contaminacion de las Aguas Continentales. Madrid: MundiPrensa, 115-157.

CURTIN, TB. \& RV LEGECKIS. 1986. Physical observation in the plume region of the Amazon River during peak discharge- I. Surface variability. Cont. Shelf Res., 6: 31-51.

DeMASTER, DJ, GB KNAPP \& CA NITTROUER. 1983. Biological uptake and accumulation of silica on the Amazon Continental Shelf. Geochim. Cosmochim. Acta, 47: 1713 - 1723.

DeMASTER, DJ, AS KUEHL \& CA NITTROUER. 1986. Effects of suspended sediments on geochemical processes near the mouth of the Amazon River: examination of biological silica uptake and the fate of particle-reactive elements. Cont. Shelf Res., 6: 107-125.

DeMASTER, DJ, WO SMITH, DM NELSON \& JY ALLER. 1996. Biogeochemical processes in Amazon shelf waters: chemical distributions and uptake rate of silicon, carbon and nitrogen. Cont. Shelf Res., 16: 617- 643.

DeMASTER, DJ. \& RH POPE. 1996. Nutrient dynamics in Amazon shelf waters: results from AMASSEDS. Cont. Shelf Res., 16: 263- 289.

DIEGUES, FMF. 1972. Introdução à Oceanografia do estuário Amazônico. In: CONGRESSO BRASILEIRO DE GEOLOGIA. Anais... São Paulo, 2: 301- 317.

FLORES MONTES, MJ, SJ MACÊDO, ML KOENING \& I LINS CORREIA. 1998. Variação nictemeral do fitoplâncton e elementos nutrientes no Canal de Santa Cruz, Itamaracá-PEBrasil. Trab. Oceanogr. Univ. Fed. Pern., 26(1): 13-26.

FOX, LE, SL SAGER \& S WOFSY. 1986. The chemical control of soluble phosphorus in the Amazon estuary. Geochim. Cosmochim. Acta, 50: 783-794.

GIBBS, RJ. 1972. Water chemistry of the Amazon River. Geochim. Cosmochim. Acta, 36: 1061 - 1066. valores de oxigênio dissolvido, $\mathrm{pH}$ e temperatura, os dados indicaram uma massa de água bem oxigenada, com valor alcalino e pequenas variações de temperatura, respectivamente.

Com base na distribuição dos nutrientes e clorofila $a$, durante as duas coletas, foi possível verificar que a transparência da água é o fator condicionante para 0 desenvolvimento da produtividade primária, na área da Plataforma Continental do Amazonas.

campo, durante a Operação Norte III (1999) e a Operação Norte IV (2001). Ao Ministério da Defesa (MD)/ Marinha do Brasil (MB), à Comissão Interministerial para os Recursos do Mar (CIRM), à Diretoria de Hidrografia e Navegação (DHN).

GEYER, RW \& GC KINEKE. 1995. Observations of currents and water properties in the Amazon frontal zone. J. Geophys. Res., 100: 2321-2339.

GRASSHOFF, K, M EHRHARDT \& K KREMLING. 1983. Methods of seawater analysis. Florida, Verlage Chemie. $417 \mathrm{p}$.

HUMBORG, C. 1997. Primary productivity regime and nutrient removal in the Danube estuary. Estuar. Coast. Mar. Sci., 45:579- 589.

LENTZ, SJ. \& R LIMEBURNER. 1995. The Amazon river plume during AMASSEDS: spatial characteristics and salinity variability. J. Geophys. Res., 100: $2355-2375$.

KINEKE, CG, WR STERNBERG, HJ TROWBRIDGE \& RW GEYER. 1996. Fluid-mud processes on the Amazon Continental Shelf. Cont. Shelf Res., 16: 667-696.

NITTROUER, CA, DJ DeMASTER, AG FIGUEIREDO \& J RINE. 1991. AMASSEDS: an interdisciplinary investigation of a complex coastal environment. Oceanog., 4: 3-7.

RYTHER, JH, DW MENZEL \& C NATHANIEL. 1967. Influence of the Amazon River outflow on the ecology of the Western Tropical Atlantic I. Hydrography and nutrient chemistry. J. of Marine Res., 25: 69- 83.

SANTOS, MLS. 2000. Influência dos rios Amazonas e Pará sobre a biomassa fitoplanctônica. Dissertação de Mestrado em Oceanografia, UFPE, Recife, PE, $180 \mathrm{p}$.

SANTOS, MLS. 2004. Distribuição dos nutrientes (Fósforo e Nitrogênio) na Plataforma Continental do Amazonas. Tese de Doutorado em Oceanografia, UFPE, Recife,PE, $136 \mathrm{p}$.

SANTOS, MLS \& AC SILVA. 2000. Estudo da temperatura, salinidade e massas d'água na plataforma continental do Amazonas e área oceânica adjacente. In: SEMANA NACIONAL DE OCEANOGRAFIA, Itajaí, SC. 432- 434.

SMITH, WOJ \& GJ RUSSELL. 1995. Phytoplankton biomass and nutrient distributions in the Amazon River plume: environmental correlates. Geo-Marine Letters., 15: 195- 198.

STRICKLAND, JDH \& TR PARSONS. 1972. A practical handbook of sea water analysis. Bull. Fish. Res. Board Can., 167: 1- 311. 
TEIXEIRA, C. 1971. Introdução aos métodos para medir a produção primária do fitoplâncton marinho. Bol. Inst. Ocean.São Paulo., 22: 59-92.

TEIXEIRA, C. \& AS GAETA. 1991. Variação nictemeral da clorofila a, produção primária do fitoplâncton e fatores ambientais da região Ubatuba (Lat. 2330'S, Long. 450' 'W). Bol. Inst. Ocean. São Paulo., 39: 15-24.

TEIXEIRA, C. \& J TUNDISI. 1967. Primary production and phytoplankton in Equatorial Waters. Bull. Mar. Sc., 17: 884- 891. UNESCO. 1973. International oceanographic table. 141 p.

Recebido: 27/04/2007

Aceito: 05/08/2010 